\title{
A Survey Study of the Dictionary Use Sub-strategies of English Majors in Saudi Arabia: Dictionary Related Aspects
}

\author{
Sultan Alhatmi ${ }^{1}$ \\ ${ }^{1}$ Department of European Languages, King Abdulaziz University, Jeddah, Saudi Arabia \\ Correspondence: Sultan Alhatmi, Department of European Languages, Faculty of Arts \& Humanities, King \\ Abdulaziz University, Jeddah, Saudi Arabia.
}

Received: January 8, 2019 Accepted: February 9, 2019 Online Published: February 11, 2019

doi: 10.5539/elt.v12n3p139 URL: https://doi.org/10.5539/elt.v12n3p139

\begin{abstract}
This study explored the sub-strategies Saudi English majors use most when consulting the dictionary. In particular, it looked at the aspects of the dictionary use strategy relevant to the dictionary itself rather than the lookup words (mainly purposes for consulting the dictionary, means of dictionary ownership and type of dictionary consulted). The participants were 90 English major students enrolled in an English undergraduate program at the Department of European Languages at King Abdulaziz University, Saudi Arabia. A survey questionnaire adapted from the literature was used to collect data for the study. The results showed that the learners' strategic preferences were largely affected by the features they liked (e.g. free dictionaries, the ease of use and search as well as portability of tech-based digital dictionaries) or disliked (e.g. the difficulty of search and use in paper dictionaries as well as their heavy weight and high thickness) most about dictionaries. Thus, they preferred to either download dictionary apps to their phones from application stores or go online whenever they needed to consult a dictionary for a word. Moreover, in terms of dictionary types, learners favored the bilingual English-Arabic dictionary (language-wise), dictionary apps and online dictionaries (medium-wise) and the ordinary dictionary (content-wise). Also, they consulted the dictionary no more than five times a day and tended to look up more words when consulting tech-based (digital) dictionaries than when using paper dictionaries. Finally, they used their dictionaries mainly to understand new words while reading.
\end{abstract}

Keywords: vocabulary learning strategy (VLS), dictionary use strategy, dictionary use sub-strategy, tech-based (digital) dictionary, dictionary app, online dictionary

\section{Introduction}

Wilkins (1972: 111) duly notes that "without grammar very little can be conveyed, without vocabulary nothing can be conveyed". Indeed, L2 learners feel that inadequate vocabulary is the reason for many problems they face in both receptive and productive language use (Nation, 1990). In this regard, research stresses that increasing recognition for the importance of explicit vocabulary training in L2 learning should be accompanied by a greater awareness of the dictionary's potential (Scholfield, 1997; Folse, 2004). McAlpine and Myles (2003: 72) neatly point out where this potential mostly lies:

Whether it is used electronically, online or in print form, its purpose is to help learners expand their vocabulary and increase their awareness of common grammar errors (Summers, 1988; Tang, 1997). In fact, it has been shown that the use of the dictionary positively affects incidental vocabulary learning, i.e. the retention of words that have been 'picked up' during listening and reading activities. An online dictionary of English phraseology that illustrates common collocations with sentence examples can help advanced learners to improve their writing skills in English and expand their passive and active vocabulary.

This study probes into the dictionary use sub-strategies (Note 1) of Saudi university learners majoring in English and its purpose is twofold: a. to survey the dictionary use habits of English majors in Saudi Arabia in order to ascertain the lookup sub-strategies that are most and least popular amongst them; b. to come up with as much comprehensible and exhaustive an inventory of dictionary use sub-strategies as possible.

\section{Significance of the Study}

Although widely seen a prominent VLS, the strategy of dictionary use received extremely little attention by researchers in Saudi Arabia. Most of the research studies found in the VLS literature which investigated 
dictionary use were mainly studies on vocabulary learning strategies that only very marginally dealt with dictionary use (e.g. Al-Qahtani, 2001; Al-Fuhaid, 2004; Al-Qahtani, 2005; Alyami, 2011). It was only recently that very few studies began to explore dictionary use independently from other VLSs (e.g. Hamouda, 2013; Alhaisoni, 2016; Al-Homoud, 2017). However, these studies surveyed the dictionary use habits and preferences of EFL learners but none were particularly directed to English majors. Moreover, they only partly covered the sub-strategies of dictionary use; whereas the questionnaire used by Hamouda (2013) included 56 items, the questionnaires employed by Al-Homoud (2017) and Alhaisoni (2016) had only 42 and 22 items respectively. As such, the significance of our study stems from the following two points:

1. It surveys the use of the dictionary strategy by English majors in particular as opposed to the other studies carried out in Saudi Arabia that investigated the wider EFL context.

2. The survey questionnaire used in the study is far more extensive as it includes 115 items covering various aspects of the strategy (Note 2).

\section{Literature Review}

\subsection{Dictionary Use Strategy: Aspects and Sub-strategies}

Asher (1999: 66) regards the use of dictionaries as a "gateway to independent learning." The crucial role of dictionary use in the language learning process has similarly been emphasized in several studies (e.g. Bensoussan, Sim, \& Weiss, 1984; Laufer, 1990; Luppescu \& Day, 1993; Tseng, 2009; Ezza \& Saadeh, 2011; Şevik, 2014). Luppescu and Day (1993) found out the students who use dictionary score significantly better in vocabulary tests than those who do not use one. Mokhtar et al. (2009) also found that Malaysian students preferred using dictionary strategies for comprehending vocabulary. Similarly, Hadavi and Hashemi (2014) concluded that, along with note-taking, dictionary strategies were the most frequently used strategies among both male and female students.

To the researcher, the common trend in the literature of talking about 'dictionary strategies' or 'dictionary use strategies' is not an accurate depiction of what really goes on every time an L2 learner uses the dictionary for help with L2 vocabulary. The use of the dictionary in itself is after all merely one of a variety of vocabulary learning strategies (VLSs) L2 learners resort to in order to facilitate the daunting task of learning the vocabulary of the foreign language (See Schmitt's (1997) VLS taxonomy for a comprehensive list of vocabulary learning strategies). A more accurate and sound term we suggest using instead for describing the various options L2 learners have during the process of dictionary use which is a strategy in and of itself is dictionary strategy 'preferences' or dictionary 'sub-strategies'. All the possible choices learners may have when using the dictionary as such should best be viewed as 'sub-strategies' or 'preferences' within a strategy rather than 'strategies' in themselves as suggested by the literature. This jargon disciplining, albeit seemingly marginal, is important in two ways. First, it helps us capture all that really goes on during dictionary use and similar macro strategies (i.e. the major vocabulary learning strategies). Second, it helps us bring more organization and discipline to the wider VLS literature by delineating the micro strategies (i.e. the minor sub-strategies) that are eventually part and parcel of the major vocabulary learning strategies.

One important goal of VLS literature is thus to capture all possible vocabulary learning strategies L2 learners may use and make inventory lists of such strategies. While these lists should be comprehensive and encompassing, they should simultaneously, for the benefit of the VLS literature, be as short and concise as possible. For example, Schmitt's (1997) taxonomy which contains a list of 57 vocabulary learning strategies, although largely good in achieving this brevity, lists monolingual dictionary and bilingual dictionary as two separate vocabulary learning strategies. Obviously, this is not in tandem with the view of conciseness of strategy wording and naming we suggest in this study. Hence, the researcher will deal with monolingual and bilingual dictionaries in this study as merely two preferences or sub-strategies within the strategy of dictionary use rather than two strategies in themselves.

Dictionary use is a very useful discovery type vocabulary learning strategy that has many aspects and sub-strategies to it. All in all, L2 learners using this strategy should deal with and make decisions regarding six main aspects of the strategy which seem to fall under two main categories:

\section{a. Dictionary related aspects:}

These are the aspects related to the dictionary itself rather than the lookup words and they include the purposes for consulting dictionary (why the learner decides to resort to dictionary), the means of dictionary ownership (how the learner gets hold of the consulted dictionary) and the type of dictionary to be consulted (what kind of dictionary is chosen for consultation). 


\section{b. Word related aspects:}

These are the aspects related to the lookup words targeted by L2 learners rather than the dictionary itself and they include source of the lookup words (where the lookup words are encountered), choice of the lookup words (how the words are selected for lookup) as well as type of word knowledge to look for (what word information is needed to fulfil a lookup).

In the following subsections, we will provide a brief overview of the dictionary related aspects as these are the ones covered in this study.

\subsubsection{Purposes for Dictionary Consultation}

Scholfield (1982a, 1999) and Nation (1990) propose that guessing from context needs to be the first step before consulting the dictionary in order to be able to pick up the appropriate meaning of a new word. However, many students turn to a dictionary whenever they meet an unknown word in their reading instead of first utilizing the techniques of guessing from context (Hadavi \& Hashemi, 2014). McCarthy (1988) stresses that many different factors affect whether or not students might turn to the dictionary for help such as how important the meaning of the word is in the passage, the number of unknown words in the text as well as the reason students are working on it.

All in all, there are the three main purposes for which L2 learners may resort to the dictionary:

a. Receptive use purposes: understand unknown words when reading and/or listening

b. Productive use purposes: use words correctly when writing and/or speaking

c. Translation purposes: translate written or spoken texts from L2 to L1 or vice versa

3.1.2 Dictionary Types: Language, Medium and Content

On the whole, learner's dictionaries may be classified in three different ways:

a. Language-wise

Depending on the language(s) included in it, an L2 learner's dictionary can be:

1. Monolingual (provides only L2 definitions)

2. Bilingual (provides only L1 definitions)

3. Bilingualized (provides both L2 alongside L1 definitions)

4. Multilingual (provides L1 definitions alongside three or more other languages)

b. Medium/Format-wise

Depending on the format or medium through which it is presented, a learner's dictionary can either come in:

1. Paper format

2. Tech-based/digital/electronic format

Tech-based dictionaries, in turn, may come in the form of a/an:

1. Portable electronic dictionary (PED)

2. Dictionary application (app)

3. CD-ROM/PC dictionary

4. Online/web/internet dictionary

c. Content-wise

Depending on their content, learner's dictionaries range from the general, ordinary multi-word information type to the more specific type such as dictionary of synonyms, dictionary of collocations and dictionary of idioms.

\subsubsection{Means of Dictionary Ownership}

To secure dictionaries for themselves, learners can either purchase, borrow or download and/or go online for dictionaries. Paper dictionaries are often purchased by L2 learners but could also be borrowed from an individual or an institutional library such as school library, university library or public library. Digital dictionaries, on the other hand, may have to be purchased but may also be available for free depending on the type of digital dictionary. Portable electronic dictionaries (PEDs), similarly to paper dictionaries, should often be purchased by the L2 learner but may well be borrowed. Dictionary apps, in turn, can be downloaded from application stores (e.g. App Store and Google Play) and may either have to be purchased by L2 learners, especially for full features 
(Pro) versions, or else made available for free by app developers and/or owners. The free versions often have fewer features than the paid ones but the basic features (e.g. providing word meaning) are included. Dictionary apps can be downloaded to the user's phone, tablet as well as computer (PC or laptop) depending on which interface is made available by developers for a particular app. CD-ROM/PC dictionaries often come free with paper dictionaries at the time of purchase but may sometimes be sold separately. Finally, online (web/internet) dictionaries are accessed free via the internet.

The first and most straightforward option for learners to obtain a dictionary is to purchase one for a fee which sometimes can be very expensive. Borrowing a dictionary, on the other hand, is an option that does not seem to be always feasible for learners for many reasons. First, some learners may feel shy to ask others to use their dictionaries for some time. Second, even for those who find no problem in making borrow requests from others, the fact that the borrowed dictionary may have to be returned at any moment to its owner and may thus not be available when really needed is a put off for borrowers. Indeed, a dictionary is a reference source that one feels more comfortable knowing that it is around and nearby all the time. Going online for online dictionaries and/or downloading free dictionary apps is, as we noted earlier, a third way L2 learners may resort to which we expect to be our subjects' favorable means for obtaining dictionaries.

With regards to digital dictionaries, the requirement of internet connectivity holds true for some of these dictionaries. Whereas a portable electronic dictionary (PED) does not require an internet connection and is available offline, a dictionary app may or may not require it and is thus available either online or offline. Like $\mathrm{PED}$, a CD-ROM/PC dictionary does not require an internet connection and is thus available offline. An online (web/internet) dictionary obviously requires an internet connection and is available only online.

\section{Research Questions}

Following the study objectives, seven research questions were posed:

RQ1. How many dictionaries do most English majors own and how many do they virtually use?

RQ2. What are the most popular purposes/reasons for which English majors consult the dictionary?

RQ3. What are the most common means (ways) through which English majors obtain the dictionaries they use?

RQ4. What are the most favorable places as well as situations in which English majors use dictionaries?

RQ5A. How often do most English majors consult the dictionary per day?

RQ5B. Do English majors look up more words when using ordinary paper dictionaries or when consulting tech-based ones?

RQ6A. What are the dictionary types most and least used by English majors across all three type categories (i.e. language-wise, medium-wise and content-wise)?

RQ6B. What is the most popular type of dictionary English majors use for translation purposes?

RQ7A. What features do English majors like most in their dictionaries?

RQ7B. What features do English majors dislike most about dictionaries?

\section{Method}

\subsection{Participants}

The participants of this study were 90 Saudi undergraduates undertaking a four-year BA in English at the Department of European Languages at King Abdulaziz University (KAU) in Jeddah, Saudi Arabia. They have already finished a preparatory year in which they took nine courses, two of which were general English courses, divided on two semesters. This foundation year aimed at preparing them for the remaining three years of a study major of their choice. The subjects were in their first year of English major study at the department and they were highly motivated to learn English.

\subsection{Instrument}

A survey questionnaire was designed to collect data for this study. Informed by the literature (namely Carstens, 1995; Kent, 2001; Nesi \& Haill, 2002; Huang, 2003; Elola, Rodríguez-García, \& Winfrey, 2009), we devised the dictionary use strategy questionnaire (DUSQ) which was intended to cover all aspects of dictionary use. In designing the DUSQ, we aimed to pinpoint all possible processes that an L2 learner in general and an English major, in particular, using a dictionary may be involved in prior to, during as well as after the lookup endeavor. To achieve this comprehensiveness in our instrument, we also referred to well-established existing models in the literature that extensively described the lookup processes and reference procedures used by L2 learners. In 
particular, we looked at Scholfield's (1982b) original model of dictionary use and reference processes employed by L2 learners. We also consulted Hartmann's (1989) strategic model of dictionary use which is an adaptation of Scholfield's model. Finally, we consulted Carstens' (1995) checklist, which she adapted from Bejoint (1989), and which in turn elaborates on Hartmann's strategic model of dictionary use.

The DUSQ covers aspects related to both the dictionary itself as well as the lookup words. Also, it covers both learners' receptive use (for listening or reading tasks) as well as productive use (for speaking or writing tasks) of the dictionary. In total, it has 115 five-point Likert scale items divided over eighteen sections. The five possible responses for each item were ( $1=$ Never, $2=$ Rarely, $3=$ Sometimes, $4=$ Often and $5=$ Always $)$ and the participants had to circle only one response that best matched their use of the dictionary sub-strategy at question. As noted earlier, due to the considerable length of the DUSQ, we administered it on two phases (the first phase covered the dictionary related aspects whereas the second phase covered the word related aspects). In this study, we only report the findings of the first phase covering the dictionary related aspects. The findings related to the second phase will be reported in a future study. For ease of reference, the first part of the DUSQ covered in this study will be referred to as the DUSQ1 whereas the second part to be covered in the later study will be referred to as the DUSQ2.

The DUSQ1 had 73 items divided over eleven sections that were related to the dictionary itself rather than the words to be looked up by learners. Table 1 shows the breakdown of the DUSQ1 items over the eleven sections.

Table 1. Breakdown of the DUSQ1 items over its eleven sections

\begin{tabular}{lll}
\hline \multicolumn{2}{l}{ Section } & Number of items \\
No. & Title & \\
\hline $\mathbf{1}$ & Likable features of dictionaries & 13 \\
$\mathbf{2}$ & Dislikable features of dictionaries & 9 \\
$\mathbf{3}$ & Reasons or purposes for dictionary use & 9 \\
$\mathbf{4}$ & Sources of dictionary used & 10 \\
$\mathbf{5}$ & Places and situations of dictionary use & 9 \\
$\mathbf{6}$ & Amount of dictionary consultation per day & 5 \\
$\mathbf{7}$ & Frequency of dictionary consultation with relevance to dictionary type & 3 \\
$\mathbf{8}$ & Type of dictionary used in terms of the language(s) used in it (language-wise) & 4 \\
$\mathbf{9}$ & Type of dictionary used in terms of its format (medium-wise) & 5 \\
$\mathbf{1 0}$ & Type of dictionary used in terms of its content (content-wise) & 3 \\
$\mathbf{1 1}$ & Type of dictionary used for translation purposes & 3 \\
Total & & $\mathbf{7 3}$ \\
\hline
\end{tabular}

The DUSQ1 was carefully translated into Arabic and its reliability has been verified using the Statistical Package for Social Sciences (SPSS) software. As Table 2 shows, the seventy-three items of the DUSQ1 were entered into SPSS and the reliability analysis showed an extremely high degree of internal item consistency with a reliability coefficient of .889. Thus, the DUSQ1 was now ready to be administered to our subjects.

Table 2. Reliability of the DUSQ1

\begin{tabular}{ll}
\hline Cronbach's Alpha & Number of items \\
\hline .889 & 73 \\
\hline
\end{tabular}

\subsection{Data Collection}

As noted earlier, the 90 students who participated in this survey study were in their first year of English major study. They belonged to three different class sections that were taught by the researcher himself. Whereas students in two of these sections were taking a writing course, students in the third section were doing a listening 
and speaking course. The Arabic version of the DUSQ1 was distributed to all 90 subjects on the same day during their three different class meetings. The students in each section were told at the beginning of class that the whole class time (1 hour and 20 minutes) will exceptionally be dedicated for a questionnaire filling session that will be held in Arabic. They were first given some background information about the study. A very brief overview of the eleven sections comprising the DUSQ1 was then provided. The students were then informed that the questionnaire filling session will be concurrent as follows:

a. The researcher would go over the items for each section of the questionnaire reading the section items out loud for them quickly explaining the items one by one and answering any questions they may raise.

b. Students would then be given some time to choose their appropriate responses for all the items of a section.

c. Once all the students finished responding to the items of a section, the procedures in a and b were repeated until the participants finished responding to all the eleven sections of the questionnaire.

The researcher preferred a concurrent questionnaire filling session rather than a regular nonconcurrent session (i.e. all students fill in the questionnaire sections at the same time rather than every student works on his own) for one reason. It would guarantee maximum follow up by the participants of the instructions and explanations provided by the researcher during the session leading to a maximum understanding of the DUSQ1 content, hence more accurate responses.

\subsection{Data Analysis}

Once the data collection finished, the researcher set out to analyze the data gathered using SPSS software. All the data obtained from the 90 questionnaires filled in by our participants was carefully entered into SPSS. Whereas percentages were used to calculate the number of dictionaries our subjects owned and/or used, mean frequency ratings were employed to measure the popularity of use for the 73 dictionary use sub-strategies included in the DUSQ1.

\section{Results and Discussion}

To answer the research question (RQ1), we counted the number of students who owned one, two, three, four or five dictionaries and the percentages for each number band were then calculated (i.e. for dictionary ownership). The same procedure was done to find out the percentages of students who virtually made use of their dictionaries (i.e. for dictionary use). To answer research questions (RQ2-RQ7B), we calculated the mean score for each of the seventy-three (73) dictionary sub-strategies identified across all strategy aspects included in the DUSQ1. Our discussion of the most and least popular dictionary sub-strategies employed by our subjects will follow the Likert scale shown in Table 3. As can be seen from the table, sub-strategies which the subjects on average reported using Always corresponded to mean scores of (4.20-5.00). Sub-strategies that were reported to be Often used corresponded to mean scores of (3.40-4.19). Others that were Sometimes used received mean scores of (2.60-3.39). Finally, whereas sub-strategies that were reported to be Rarely used had mean scores of (1.80-2.59), those that were Never used equaled mean scores of (1.00-1.79).

Table 3. Sub-strategy mean score range and corresponding Likert scale equivalent

\begin{tabular}{ll}
\hline Mean score range & Likert scale equivalent \\
\hline $1.00-1.79$ & Never \\
$1.80-2.59$ & Rarely \\
$2.60-3.39$ & Sometimes \\
$3.40-4.19$ & Often \\
$4.20-5.00$ & Always \\
\hline
\end{tabular}

\section{RQ1. How many dictionaries do most English majors own and how many do they virtually use?}

Whereas most of our subjects (45.6\%) reported ownership of two dictionaries, $37.8 \%$ reported having only one dictionary. Only very few subjects reported they owned three (12.2\%) or four (4.4\%) dictionaries (see Table 4 and Figure 1). With regards to actual dictionary use, the vast majority of our subjects (68.9\%) reported the use of only one of the dictionaries they owned. This is likely to come after trying one or more of the dictionaries they reported owning and then sticking to the one that they felt more comfortable with. Whereas $27.8 \%$ of our subjects reported using two dictionaries, only 3.3\% reported the use of three dictionaries perhaps to meet word 
lookup tasks of more variety and volume for which the use of one or even two dictionaries may not have been sufficient to meet (see Table 5 and Figure 2).

Table 4. Number of dictionaries owned by learners

\begin{tabular}{lll}
\hline Number of dictionaries & Frequency & Percent \\
\hline one dictionary & 34 & 37.78 \\
two dictionaries & 41 & 45.56 \\
three dictionaries & 11 & 12.22 \\
four dictionaries & 4 & 4.44 \\
Total & 90 & 100 \\
\hline
\end{tabular}

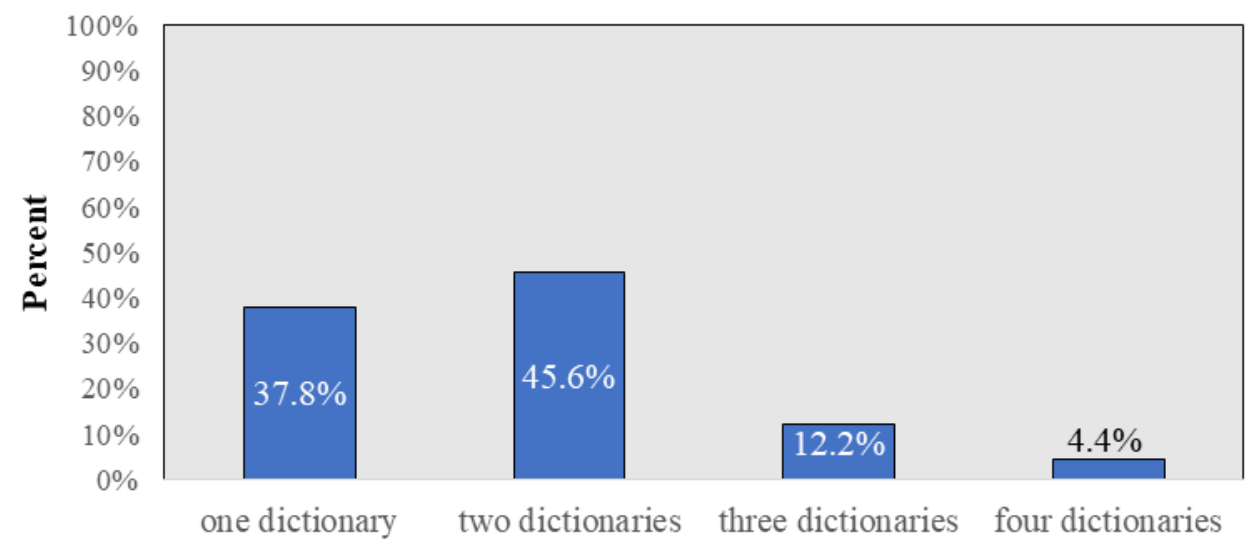

Figure 1. Number of dictionaries owned by leaners

Table 5. Number of dictionaries used by learners

\begin{tabular}{lll}
\hline Number of dictionaries & Frequency & Percent \\
\hline one dictionary & 62 & 68.89 \\
two dictionaries & 25 & 27.78 \\
three dictionaries & 3 & 3.33 \\
Total & 90 & 100 \\
\hline
\end{tabular}

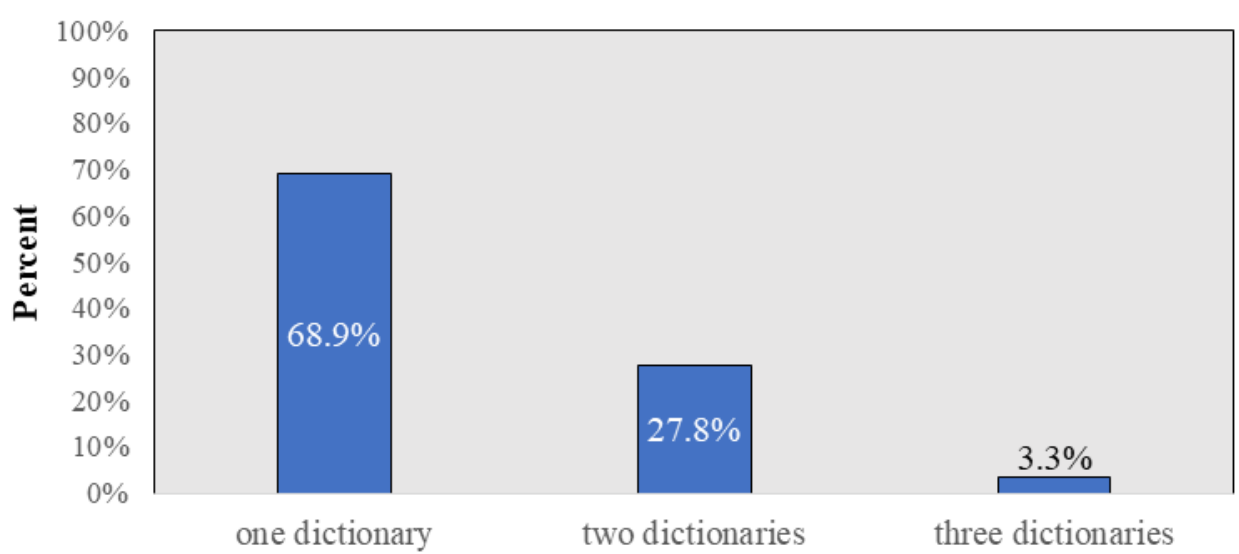

Figure 2. Number of dictionaries used by leaners 


\section{RQ2. What are the most popular purposes/reasons for which English majors consult the dictionary?}

In general, most of the purposes/reasons for dictionary use seemed equally valid for our subjects. This is evident from the very close mean scores reported for these purposes/reasons. Nevertheless, 'using the dictionary to understand new words while reading' was reported by far the most popular purpose/reason for which our subjects consulted the dictionary (mean=4.47). Indeed, reading comprehension is an essential component of L2 learning and the use of the dictionary strategy for many EFL learners seems an indispensable facilitating aid to comprehension while reading. As noted earlier, the remaining purposes/reasons had very close mean scores ranging from 3.06 to 3.87 which indicates that these were equally important purposes/reasons for our subjects for using the dictionary strategy. Finally, it can be noted that using the dictionary to translate written or spoken texts from English to Arabic was far more common than from Arabic to English (means= 3.40 and 2.60 respectively). This is expected since our subjects are English majors and thus most of the texts they encounter are in English (see Table 6 and Figure 3).

Table 6. Reasons/purposes for dictionary use

\begin{tabular}{llll}
\hline Rank & Reason/purpose & Mean & SD \\
\hline $\mathbf{1}$ & Understand words I do not know when reading & 4.47 & .91 \\
$\mathbf{2}$ & Use words correctly when writing & 3.87 & 1.23 \\
$\mathbf{3}$ & Understand words I do not know when listening & 3.83 & 1.23 \\
$\mathbf{4}$ & Assignment for class & 3.74 & 1.15 \\
$\mathbf{5}$ & Use words correctly when speaking & 3.62 & 1.43 \\
$\mathbf{6}$ & Know meanings of contextual words or phrases & 3.52 & 1.24 \\
$\mathbf{7}$ & Translate written or spoken texts from English to Arabic & 3.40 & 1.44 \\
$\mathbf{8}$ & Know meanings of separate/ isolated words or phrases & 3.06 & 1.40 \\
$\mathbf{9}$ & Translate written or spoken texts from Arabic to English & 2.60 & 1.40 \\
\hline
\end{tabular}

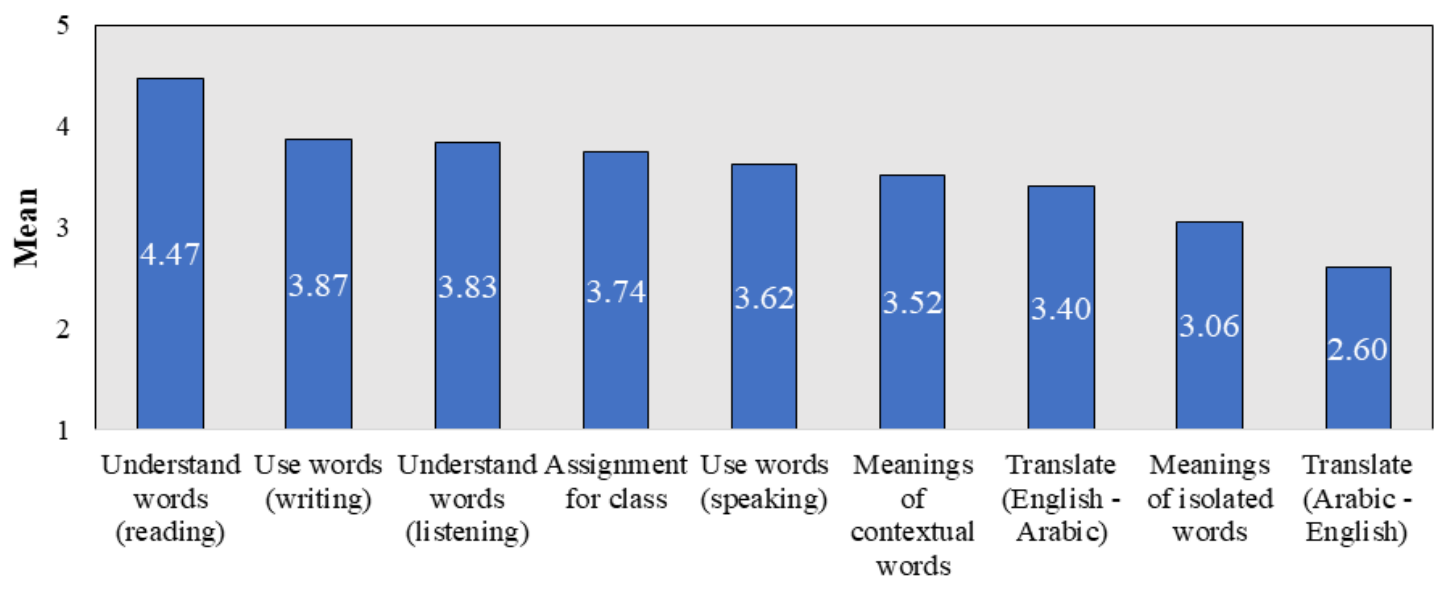

Figure 3. Reasons/ purposes for dictionary use

\section{RQ3. What are the most common means through which English majors obtain the dictionaries they use?}

Downloading dictionary apps from application stores was by far the most popular way through which our subjects obtained their dictionaries (mean= 4.47). Going online to consult online dictionaries for word information ranked second (mean=3.54). It should be noted that our subjects preferred to download dictionary apps from app stores to their phones (mean=4.47) considerably more often than to their tablets or computers $(m e a n=2.20)$. That is, they rarely downloaded dictionary apps to a tablet or computer and always preferred to download their apps to a mobile phone instead. This is most likely due to the fact that mobile phones have more portability (e.g. they can go into one's pocket) than tablets and computers which makes it easier for learners to use their dictionary apps right from their 'little' devices rather than the 'bigger' ones. 
CD-ROM dictionary, on average, was reported to be never used by our subjects (mean=1.34). This is most likely because a CD-ROM dictionary often comes with a purchased paper dictionary, a preference which our subjects reported to extremely rarely resort to in the first place (mean=1.89). Another possible reason is that not all paper dictionaries when purchased are accompanied with CD-ROM copies. A third reason that may well deter English majors from using CD-ROM dictionaries is that, compared to the other tech-based dictionaries (namely dictionary app and online dictionary), CD-ROM dictionary is far less handy and manageable. This is because whereas a dictionary app and an online dictionary can literally work on any device, a CD-ROM dictionary always requires a computer (laptop or PC) that is equipped with a CD-ROM drive facility.

Finally, our subjects reported no interest in borrowing dictionaries from friends or university library. This is likely because dictionary ownership in case of borrowing is not feasible. That is, a dictionary is essentially a reference source that a learner resorts to repeatedly and thus would like to see around all the time which cannot be the case if it was borrowed from someone else (see Table 7 and Figure 4).

Table 7. Means of dictionary ownership

\begin{tabular}{llll}
\hline Rank & Means/ Way & Mean & SD \\
\hline $\mathbf{1}$ & Downloaded dictionary app to my phone from an application store & 4.47 & 1.13 \\
$\mathbf{2}$ & Go online when I need to consult a dictionary for a word & 3.54 & 1.49 \\
$\mathbf{3}$ & Downloaded dictionary app to my tablet/computer from an application store & 2.20 & 1.66 \\
$\mathbf{4}$ & Purchased paper dictionary myself & 1.89 & 1.48 \\
$\mathbf{5}$ & Received a CD copy of my paper dictionary when I bought it & 1.34 & 1.05 \\
$\mathbf{6}$ & Purchased portable electronic dictionary (PED) myself & 1.21 & .81 \\
$\mathbf{7}$ & Borrowed paper dictionary from university library & 1.18 & .57 \\
$\mathbf{8}$ & Borrowed paper dictionary from a friend & 1.18 & .46 \\
$\mathbf{9}$ & Borrowed portable electronic dictionary (PED) from a friend & 1.16 & .63 \\
$\mathbf{1 0}$ & Borrowed portable electronic dictionary (PED) from university library & 1.02 & .21 \\
\hline
\end{tabular}

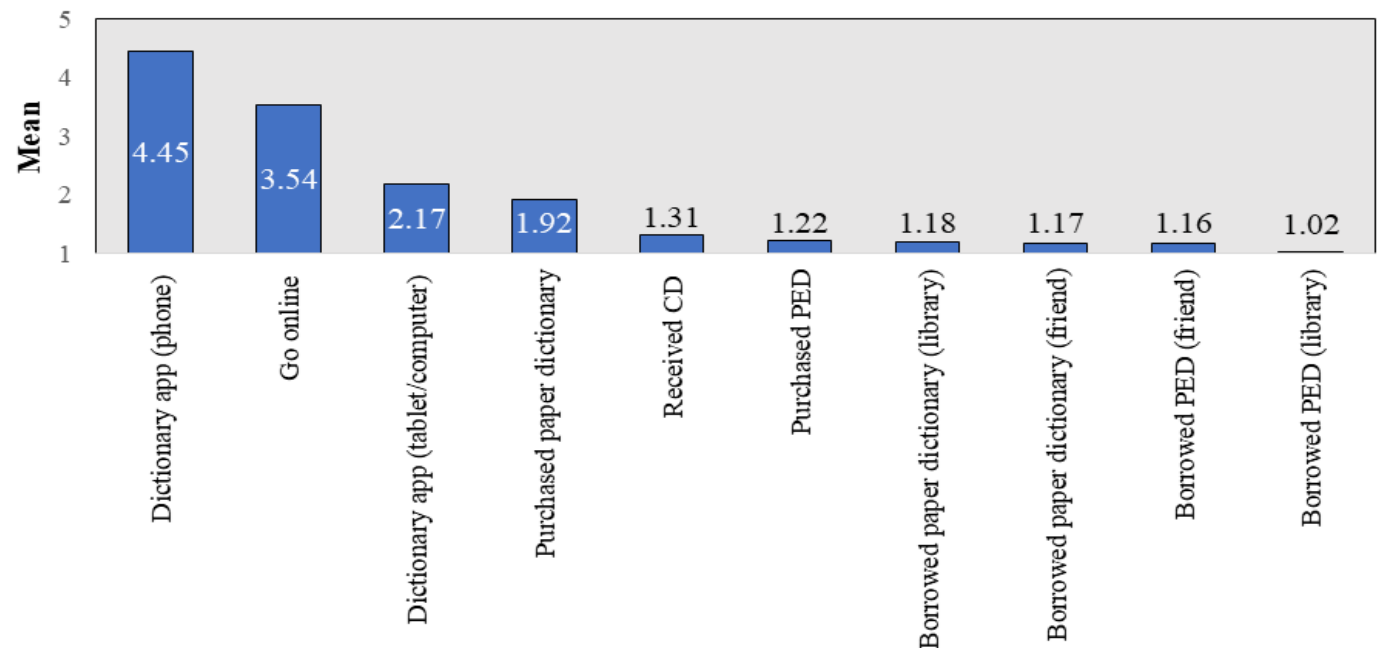

Figure 4. Means of dictionary ownership

\section{RQ4. What are the most favorable places as well as situations in which English majors use dictionaries?}

Home was the most favorable place our subjects reported using their dictionaries at (mean=4.48). This was expected since most of any student's actual study takes place at home. University ranked the second most favorable place for dictionary lookup (mean= 3.79). Thus, the limited time of 'dictionary contact', so to speak, available for English majors at university due to their engagement with running classes can indeed be best 
compensated for at home. Learners virtually have considerably more time at home to look up new words and even revisit the ones they looked up at university. All other places were reported by our subjects to be rarely used for dictionary lookup (see Table 8 and Figure 5).

Table 8. Places of dictionary use

\begin{tabular}{llll}
\hline Rank & Place & Mean & SD \\
\hline $\mathbf{1}$ & At home & 4.48 & .86 \\
$\mathbf{2}$ & At university & 3.79 & 1.16 \\
$\mathbf{3}$ & At other places & 2.62 & 1.20 \\
$\mathbf{4}$ & In the library & 1.98 & 1.30 \\
$\mathbf{5}$ & When travelling from home to school or vice versa & 1.96 & 1.26 \\
\hline
\end{tabular}

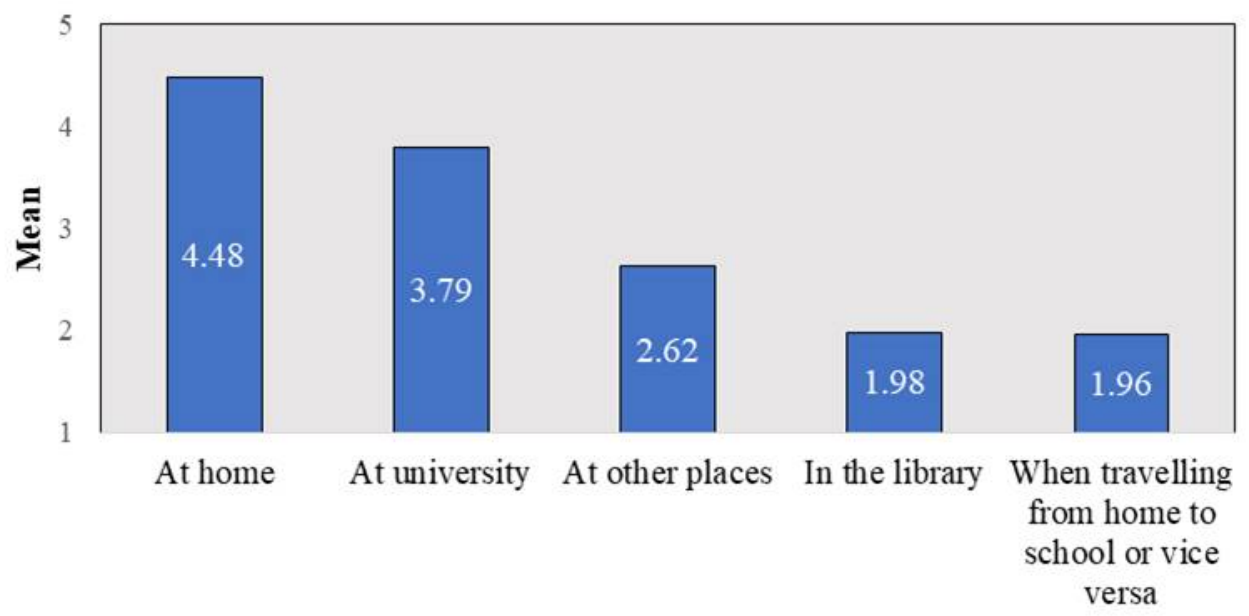

Figure 5. Places of dictionary use

As for the situations of dictionary use, our subjects reported using the dictionary predominantly when studying for their basic English courses (i.e. listening, speaking, reading and writing) (mean=4.28). This is no wonder since these basic skills courses essentially represent the very first encounters with the L2 for any English major student in his/her formal journey of L2 learning. Thus, the dictionary seems an indispensable tool for them at this early stage of their formal specialized study of English. In fact, our subjects were in their first year of study in which they only had these basic skills courses but had none of the advanced courses (i.e. literature, linguistics and translation) yet, hence the extremely low mean scores for dictionary use associated with these advanced courses $(1.33,1.31$ and 1.24 respectively) which indicate that no use of the dictionary was associated with these courses. Had our subjects been in their third or fourth year, we might have seen some use of the dictionary associated with these courses so as to deal with the new words and terminology often encountered in the literature and textbooks of such advanced courses (see Table 9 and Figure 6).

Table 9. Situations of dictionary use

\begin{tabular}{llll}
\hline Rank & Situation & Mean & SD \\
\hline $\mathbf{1}$ & For my basic English courses at university (listening, speaking, reading, writing) & 4.28 & 1.06 \\
$\mathbf{2}$ & For my other advanced English courses at university (namely literature courses) & 1.33 & .99 \\
$\mathbf{3}$ & For my advanced English courses at university (namely linguistics courses) & 1.31 & 1.00 \\
$\mathbf{4}$ & For my translation courses at university & 1.24 & .84 \\
\hline
\end{tabular}




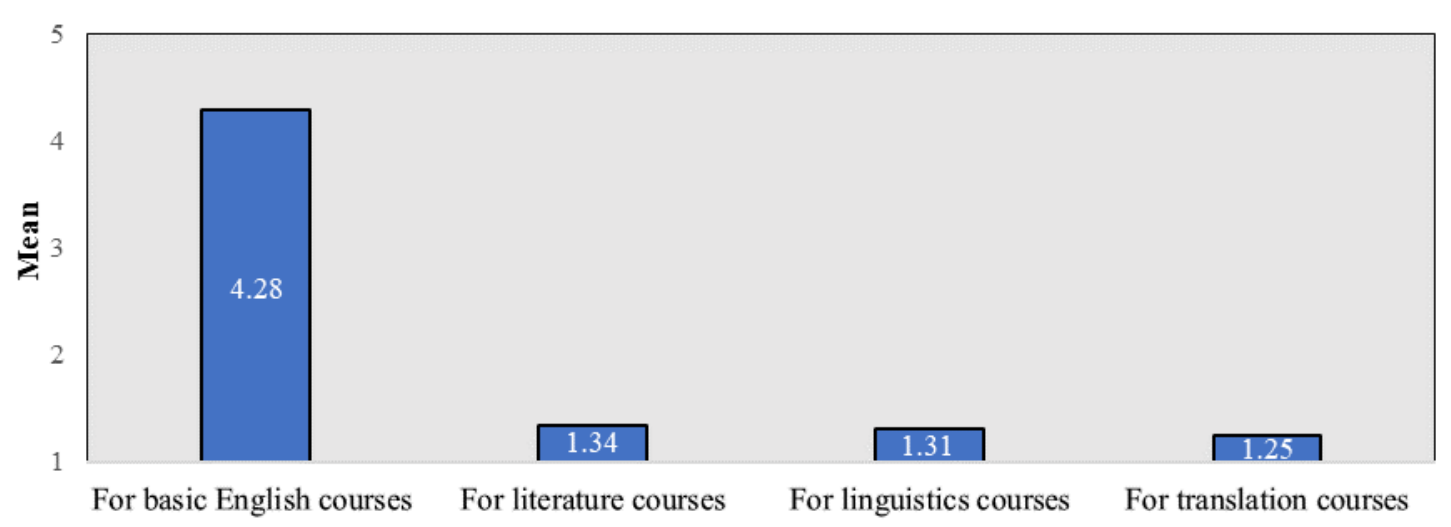

Figure 6. Situations of dictionary use

\section{RQ5A. How often do most English majors consult the dictionary per day?}

Generally speaking, the mean scores for the different frequency bands of dictionary consultation per day reported by our subjects show an overall moderate use of the dictionary strategy with the mean for the 1-5 times band as the highest of all bands with 2.70 . Whereas our subjects reported that they would sometimes consult their dictionaries from one to five times a day (mean=2.70), they would rarely use the dictionary five to ten times per day (mean=1.93). Finally, our subjects reported that they would never consult the dictionary more than ten times a day as evident from the mean scores of the remaining three frequency bands (means=1.32, 1.19 and 1.15). As far as the researcher is concerned, this moderate to low use of the strategy may be attributed to two reasons. First, some learners may be deterred from using the strategy simply due to possibly lacking the skills necessary to use the dictionary. Also, other learners may well be engaged in other vocabulary learning strategies that are more favorable and perhaps easier than using the dictionary (e.g. asking teachers or classmates for word information). Thus, this possible dependency on other common strategies for uncovering word information would certainly minimize the learners' use of the dictionary strategy resulting in fewer consultations of the dictionary per day.

At any rate, it should be noted here that we chose to use the 'per day' over the 'per week' frequency bands for assessing our subjects' frequency of dictionary use as it is obviously easier for one to remember and report what they do on a single day than over a whole week. Thus, more accurate responses in this relevance could be elicited from the subjects (see Table 10 and Figure 7).

Table 10. Frequency of dictionary consultation per day

\begin{tabular}{llll}
\hline Rank & Frequency band & Mean & SD \\
\hline $\mathbf{1}$ & $1-5$ times a day & 2.70 & 1.55 \\
$\mathbf{2}$ & $5-10$ times a day & 1.93 & 1.27 \\
$\mathbf{3}$ & $10-15$ times a day & 1.32 & .67 \\
$\mathbf{4}$ & $15-20$ times a day & 1.19 & .50 \\
$\mathbf{5}$ & More than 20 times a day & 1.15 & .44 \\
\hline
\end{tabular}




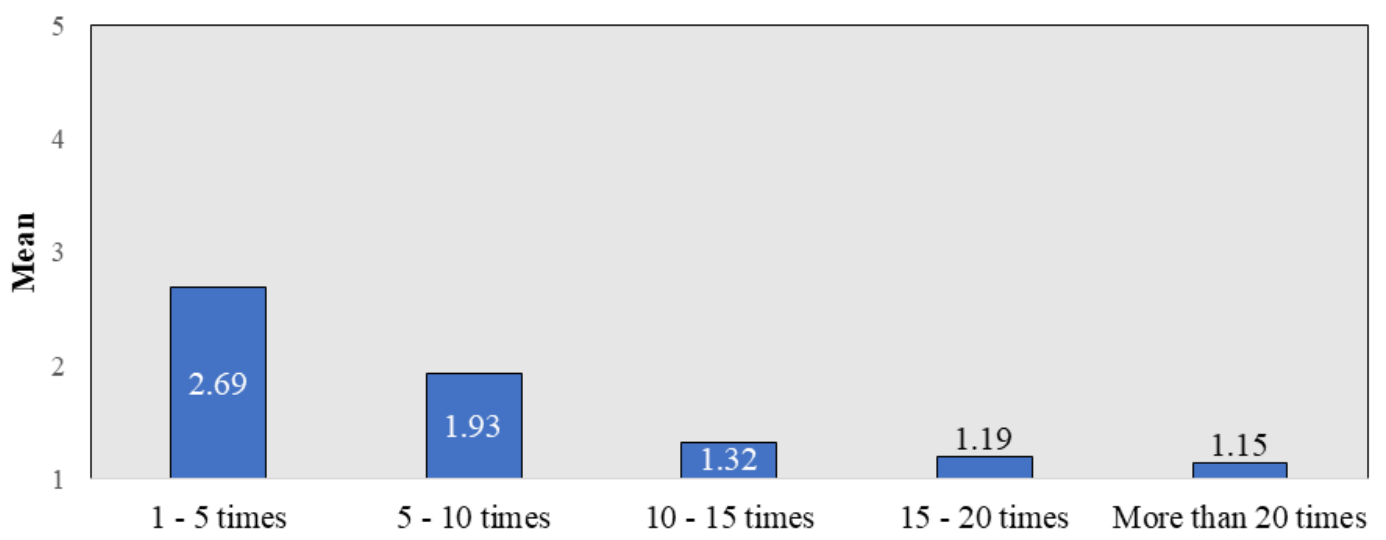

Figure 7. Frequency of dictionary consultation per day

\section{RQ5B. Do English majors look up more words when using ordinary paper dictionaries or when consulting tech-based ones?}

Our subjects reported that they often look up more words when consulting tech-based dictionaries as opposed to ordinary paper dictionaries (mean=3.97). On the contrary, they reported that they would almost never look up more words when consulting ordinary paper dictionaries compared to tech-based dictionaries (mean=1.42). The mean score in response to the item 'type of dictionary (tech-based or paper) does not make a difference for me when it comes to the number of words I will be looking up' was extremely low (mean=1.62) indicating that they were indeed inclined to one type over the other as we just found. This result is in line with the learners' reported extreme preference of using dictionary apps and online dictionaries over ordinary paper dictionaries (see the answer to RQ6A). It also comes as no surprise since they reported an immensely high liking for tech-based dictionaries either for the ease of use and search in them, for their portability as well as for the speaking function embedded in them. By the same token, our subjects reported a very low liking for paper dictionaries even ones that had good paper and print quality (see the answer to RQ7A). They also reported an immense dislike for the difficulty of search and use in paper dictionaries as well as their heavy weight and high thickness (see the answer to RQ7B).

This outright preference of our subjects for tech-based dictionaries over paper dictionaries may partly be attributed to the lookup speed of digital dictionaries as they can deliver word knowledge considerably faster than paper dictionaries. In this regard, Weschler and Pitts (2000) compared students' lookup speed of electronic and paper dictionary. They took no account of the quality or number of definitions or even the students' ability to read and comprehend them. They found that the average lookup time for ten words using a paper dictionary was 168 seconds (about 17 seconds per word) and using an electronic dictionary 130 seconds (about 13 seconds per word). That is, their students could look up words with an electronic dictionary about $23 \%$ faster than with a paper dictionary (see Table 11 and Figure 8).

Table 11. Frequency of dictionary consultation and dictionary type

\begin{tabular}{llll}
\hline Rank & Frequency and dictionary type & Mean & SD \\
\hline $\mathbf{1}$ & More words tech-based dictionaries & 3.97 & 1.41 \\
$\mathbf{2}$ & Type of dictionary does not make a difference & 1.62 & 1.06 \\
$\mathbf{3}$ & More words ordinary paper dictionaries & 1.42 & .94 \\
\hline
\end{tabular}




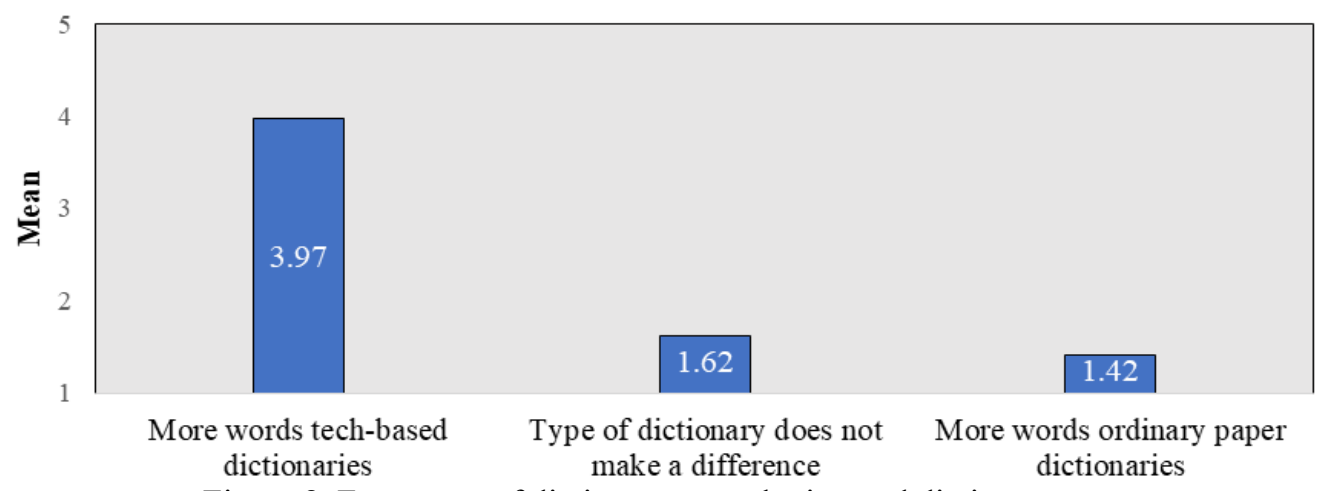

Figure 8 . Frequency of dictionary consultation and dictionary type

\section{RQ6A. What are the dictionary types most and least used by English majors across all three type categories (i.e. language-wise, medium-wise and content-wise)?}

In terms of languages used in the dictionary (i.e. language-wise), the bilingual English-Arabic dictionary was the type most used by our subjects (mean= 3.74). The bilingual Arabic-English dictionary was far less favorable (mean= 2.63). This is no wonder given the fact that our subjects were English majors whose language of instruction was English and whose textbooks were entirely in English. Thus, English (L2) was essentially the starting point for all their learning situations which was bound to be reflected in their choice of bilingual dictionary (i.e. L2 to L1 rather than the other way around).

The monolingual English-English dictionary ranked second in popularity amongst our subjects (mean=3.08). The learners' preference of the bilingual English-Arabic over the monolingual dictionary is most likely due to the less time and effort it involves for word lookups. Indeed, the bilingual L2 to L1 dictionary is faster and easier to use than a monolingual dictionary especially for beginning learners such as ours. Thus, given that our subjects were first-year English majors who were in their very first semester in the department after finishing the foundation year, they generally had lower proficiency levels in English compared to advanced third or fourth-year students. Consequently, their vocabulary proficiency level in the L2 was still low at this early stage of L2 learning making it difficult for them to decode the meaning of new words via the L2 itself. Thus, they often resorted to the L1 as a medium for decoding the meaning of new L2 words, hence the more use of the bilingual English-Arabic dictionary.

By the same token, since the bilingualized English-English-Arabic dictionary puts the L2 as another medium along with the L1 for decoding meanings of new L2 words, it certainly slows down the lookup process and adds more complication to it. As such, our subjects almost avoided using the bilingualized dictionary for their lookup endeavors (mean=1.98) (see Table 12 and Figure 9).

Table 12. Type of dictionary used in terms of the language(s) used in it (language-wise)

\begin{tabular}{llll}
\hline Rank & Type of dictionary (language-wise) & Mean & SD \\
\hline $\mathbf{1}$ & Bilingual (English-Arabic) & 3.74 & 1.32 \\
$\mathbf{2}$ & Monolingual (English-English) & 3.08 & 1.34 \\
$\mathbf{3}$ & Bilingual (Arabic-English) & 2.63 & 1.55 \\
$\mathbf{4}$ & Bilingualized (English-English-Arabic) & 1.98 & 1.37 \\
\hline
\end{tabular}




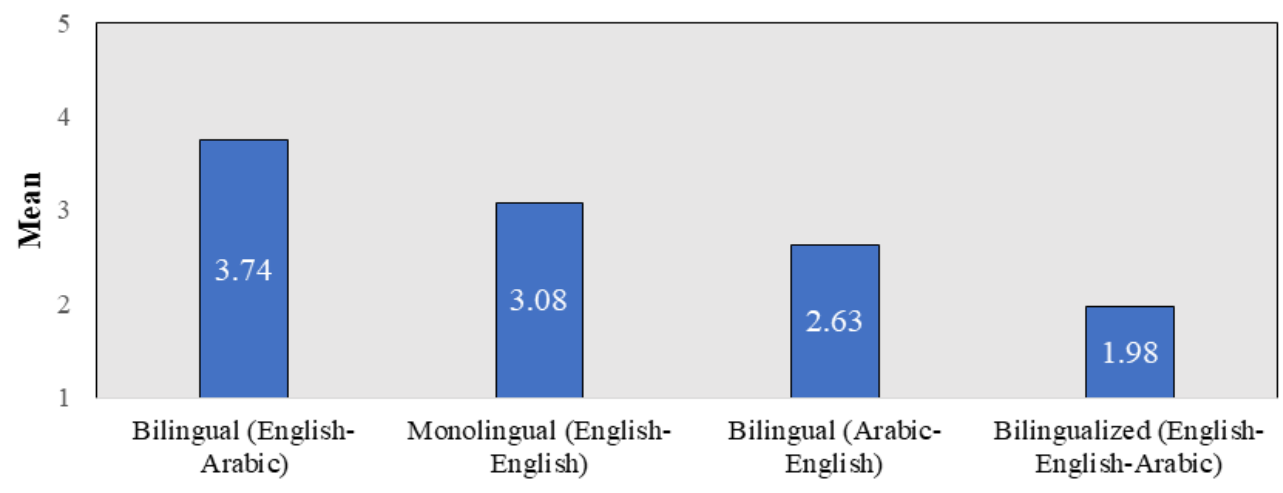

Figure 9. Type of dictionary used (language-wise)

As for dictionary types in terms of format (i.e. medium-wise), dictionary app and online dictionary were the most popular types our subjects reported using (means $=4.29$ and 3.48 respectively). On the contrary, paper dictionary, CD-ROM dictionary and portable electronic dictionary (PED) were very unpopular (means= 1.74 , 1.21 and 1.20 respectively). Again, as we noted earlier, the dictionary features that our subjects reported to like (see the answer to RQ7A) and/or dislike (see the answer to RQ7B) seem to be well reflected in their preferences for dictionary type. First and foremost, whereas paper dictionary, CD-ROM dictionary and portable electronic dictionary (PED) are not free, dictionary apps and online dictionaries are often accessed free of charge. Secondly, dictionary apps and online dictionaries are far easier to use and search. Thirdly, they are extremely more portable compared to the other three dictionary medium types. Whereas a dictionary app and online dictionary do not really have physical existence (i.e. they are merely viewable through computerized devices), paper dictionary, CD-ROM dictionary and portable electronic dictionary (PED) all physically exist. Fourth, a dictionary app and an online dictionary have a speaking function embedded in them (i.e. they can virtually pronounce words), a feature which a paper dictionary obviously cannot provide (see Table 13 and Figure 10).

Table 13. Type of dictionary used in terms of its format (medium-wise)

\begin{tabular}{llll}
\hline Rank & Type of dictionary (medium-wise) & Mean & SD \\
\hline $\mathbf{1}$ & Dictionary app & 4.29 & 1.16 \\
$\mathbf{2}$ & Online dictionary & 3.48 & 1.37 \\
$\mathbf{3}$ & Paper dictionary & 1.74 & .92 \\
$\mathbf{4}$ & CD-ROM dictionary & 1.21 & .71 \\
$\mathbf{5}$ & Portable electronic dictionary (PED) & 1.20 & .60 \\
\hline
\end{tabular}

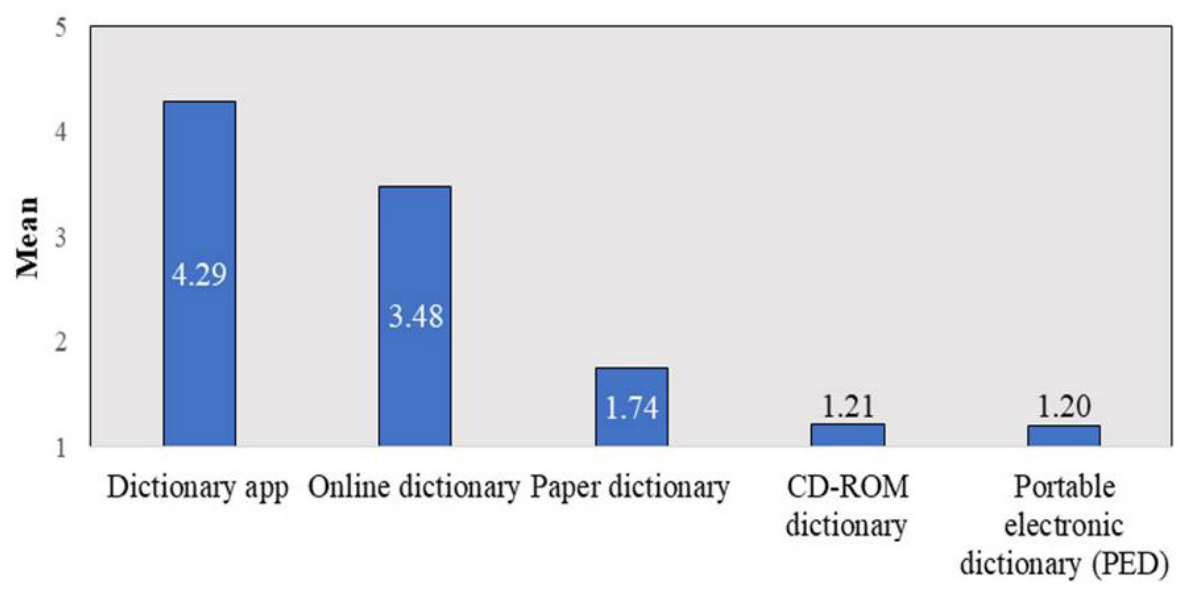

Figure 10. Type of dictionary used (medium-wise) 
As for dictionary types in terms of content (i.e. content-wise), the ordinary dictionary was the most popular type our subjects used (mean=4.13). Dictionary of synonyms and dictionary of collocations/idioms were rarely used (means $=2.14$ and 1.83 respectively). This could simply be due to these two types being:

a. extremely infamous amongst L2 learners as it seems that only very few learners know of them;

b. not always available as they rarely exist either in print form or tech-based form (app, CD, online) with the proportion of ordinary dictionaries far outweighing that of specific content types.

In parallel, the ordinary dictionary, which is known for containing a variety of word information for each word entry, is very well known amongst L2 learners and exist in abundance either in print form or tech-based form (app, CD, online) (see Table 14 and Figure 11).

Table 14. Type of dictionary used in terms of its content (content-wise)

\begin{tabular}{llll}
\hline Rank & Type of dictionary (content-wise) & Mean & SD \\
\hline $\mathbf{1}$ & Ordinary dictionary & 4.13 & 1.05 \\
$\mathbf{2}$ & Dictionary of synonyms & 2.14 & 1.29 \\
$\mathbf{3}$ & Dictionary of collocations/idioms & 1.83 & 1.05 \\
\hline
\end{tabular}

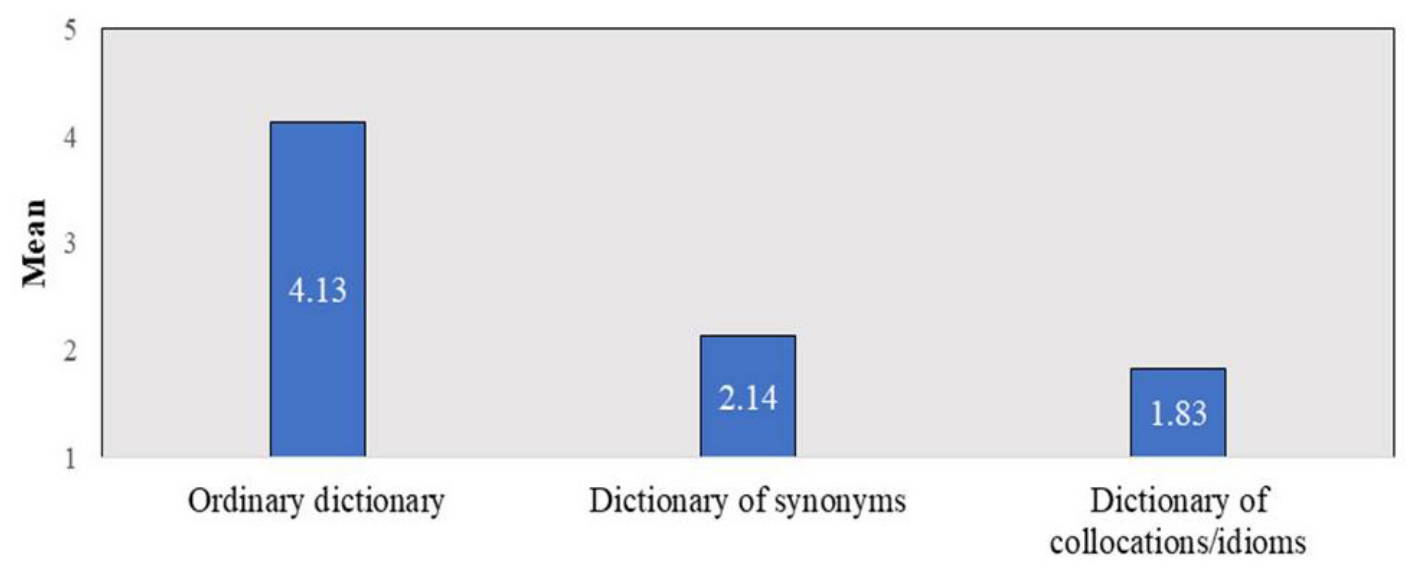

Figure 11. Type of dictionary used (content-wise)

\section{RQ6B. What is the most popular type of dictionary English majors use for translation purposes?}

Again, our subjects' preferences within the three types of bilingual dictionaries for general dictionary purposes/ uses resembled those for translation purposes. Thus, the English-Arabic dictionary was the type they used most for translation purposes (mean=4.07). The Arabic-English dictionary was less favorable (mean=3.20). Since our subjects were English majors (i.e. their target language was English and the texts they encountered were all in English), the starting language for most translations they needed to carry out had to be the L2 (English) rather than the L1 (Arabic), hence their preference for the English-Arabic dictionary. The bilingualized English-English-Arabic dictionary would, as is the case with general dictionary use, add more burden to a translation task as it involves using the target language as a mediator between the target language item to be translated (i.e. word /phrase/idiom) and the sought L1 equivalent, something that will undoubtedly slow the translation process. Therefore, our subjects tended to avoid this type of dictionary for their translation purposes $($ mean=2.40) (see Table 15 and Figure 12). 
Table 15. Type of dictionary used for translation purposes

\begin{tabular}{llll}
\hline Rank & Type of dictionary & Mean & SD \\
\hline $\mathbf{1}$ & English-Arabic dictionary & 4.07 & 1.27 \\
$\mathbf{2}$ & Arabic-English dictionary & 3.20 & 1.52 \\
$\mathbf{3}$ & English- English-Arabic dictionary & 2.40 & 1.51 \\
\hline
\end{tabular}

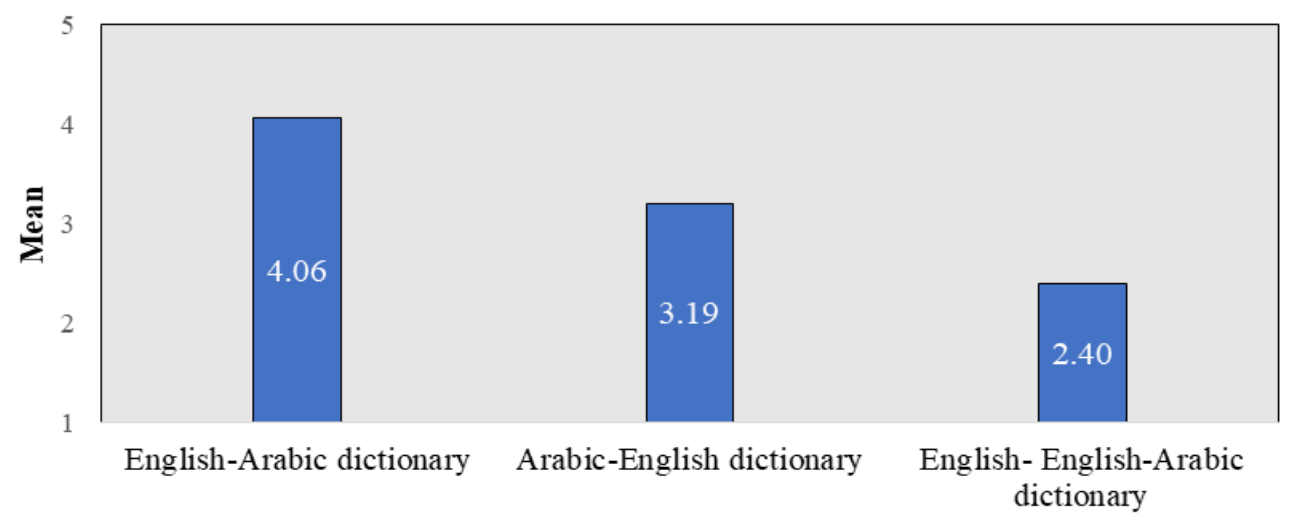

Figure 12. Type of dictionary used for translation purposes

\section{RQ7A. What features do English majors like most in their dictionaries?}

Being free of charge was reported by our subjects the most likable feature in a dictionary (mean=4.53). This is certainly one reason why they also enormously liked online dictionaries since they are essentially free (mean= 4.44). The ease of use and search in tech-based dictionaries was also highly appreciated (mean=4.32). Similarly, the great portability characteristic of digital dictionaries, as well as the speaking function embedded in them, were immensely admired by our subjects (means $=4.22$ and 4.15 respectively). Other dictionary features which had high likeability to our subjects included having example sentences for headwords (mean=3.91), containing Arabic translation for them (mean $=3.76$ ) as well as having a large number of headwords (mean=3.74).

A closer look into the findings of this study (in particular see answers to RQ3, RQ5B, and RQ6A) shows that the dictionary features that our subjects reported to like and dislike most seem to have plausibly affected their preferences on some aspects of the dictionary use strategy. The following are the instances we spotted of the above-stated link between the dictionary features most likable to our subjects and their strategic preferences as to dictionary use:

\section{1). Free dictionaries}

- With relevance to means of dictionary ownership (RQ3), the two most common ways through which our subjects obtained their dictionaries were 'downloading dictionary app to my phone from an application store' as well as 'going online when I need to consult a dictionary". Unlike PEDs and paper dictionaries which are paid and often expensive, these two means of dictionary ownership are often free of cost.

- With relevance to dictionary format (RQ6A), dictionary apps (most are free) and online dictionaries (all are free) were reported as the two most commonly used format types. Whereas these formats are available often free, pocket electronic dictionaries (PEDs) and paper dictionaries are paid and often expensive and thus they were almost never used by our subjects.

\section{2). Tech-based dictionaries are easy to use and search}

- With relevance to means of dictionary ownership (RQ3), again, the two most common sources for the learners' dictionaries were 'downloading dictionary app to my phone from an application store' as well as 'going online when I need to consult a dictionary". Unlike paper dictionaries which are traditional and difficult to use and search, these two means of dictionary ownership are based on technology and are thus far easier to use and search. 
- With relevance to frequency of dictionary consultation and dictionary type (RQ5B), our subjects reported looking up more words in tech-based dictionaries as opposed to the more difficult to use and search paper dictionaries.

- With relevance to dictionary format (RQ6A), dictionary apps and online dictionaries were reported as the two most commonly used formats. These formats are based on technology and are far easier to use and search than the traditional paper dictionaries which were very unpopular.

\section{3). Tech-based dictionaries are portable}

- With relevance to means of dictionary ownership (RQ3), again the two most common sources for the learners' dictionaries were 'downloading dictionary app to my phone from an application store' as well as 'going online when I need to consult a dictionary". Unlike paper dictionaries which are less portable and more difficult to carry, these two means of dictionary ownership are clearly more portable and easier to carry.

- With relevance to frequency of dictionary consultation and dictionary type (RQ5B), our subjects reported looking up more words in tech-based dictionaries as opposed to the less portable and more difficult to carry paper dictionaries.

- With relevance to dictionary format (RQ6A), dictionary apps and online dictionaries were reported as the two most commonly used formats. These formats are clearly more portable and easier to carry than paper dictionaries.

\section{4). Speaking function in tech-based dictionaries}

- With relevance to means of dictionary ownership (RQ3), again the two most common sources for the learners' dictionaries were 'downloading dictionary app to my phone from an application store' as well as 'going online when I need to consult a dictionary". Unlike paper dictionaries which lack the speaking function, these two means of dictionary ownership have the speaking function embedded in them by default.

- With relevance to frequency of dictionary consultation and dictionary type (RQ5B), our subjects reported looking up more words in tech-based dictionaries as opposed to paper dictionaries which obviously lack the speaking function.

- With relevance to dictionary format (RQ6A), dictionary apps and online dictionaries were reported as the two most commonly used formats. Whereas these two formats have a speaking function embedded in them by default, paper dictionaries obviously lack this function.

\section{5). Arabic translation for words}

- With relevance to dictionary language (RQ6A), the bilingual English-Arabic dictionary was reported the most commonly used as opposed to the monolingual English-English dictionary which obviously does not provide Arabic equivalents for looked up words.

\section{6). A large number of headwords}

- With relevance to dictionary content (RQ6A), the ordinary dictionary was reported the most commonly used type. Whereas the ordinary dictionary has a large number of headwords (word entries), a dictionary of synonyms and a dictionary of collocations/idioms often have a smaller number of headwords.

Finally, our subjects have not shown much interest in dictionaries that are cheap (mean= 2.73). This is most likely because, as we have seen, they have shown an outright likeability for free dictionaries which resulted in them having less interest in chargeable dictionaries in the first place even cheap ones. In other words, they were interested in dictionaries that were totally free of charge. Similarly, paper dictionaries, even ones with good paper print and quality, did not attract our subjects (mean=2.17) which is in line with their reported low use of them as we have seen in the answer to RQ6A (see Table 16 and Figure 13). 
Table 16. Likable features of dictionaries

\begin{tabular}{llll}
\hline Rank & Feature & Mean & SD \\
\hline $\mathbf{1}$ & Free dictionaries & 4.53 & .93 \\
$\mathbf{2}$ & Online dictionaries are free & 4.44 & .94 \\
$\mathbf{3}$ & Tech-based dictionaries are easy to use and search & 4.32 & 1.26 \\
$\mathbf{4}$ & Tech-based dictionaries are portable & 4.22 & 1.38 \\
$\mathbf{5}$ & Speaking function in tech-based dictionaries & 4.15 & 1.28 \\
$\mathbf{6}$ & Example sentences for words & 3.91 & 1.24 \\
$\mathbf{7}$ & Arabic translation for words & 3.76 & 1.34 \\
$\mathbf{8}$ & A large number of headwords & 3.74 & 1.43 \\
$\mathbf{9}$ & A nice layout & 3.22 & 1.54 \\
$\mathbf{1 0}$ & Word entries, terms, collocations, idioms etc. are colored & 3.20 & 1.48 \\
$\mathbf{1 1}$ & Illustrations/pictures for words & 3.01 & 1.54 \\
$\mathbf{1 2}$ & Cheap dictionaries & 2.73 & 1.61 \\
$\mathbf{1 3}$ & Paper dictionary that has good paper and print quality & 2.17 & 1.50 \\
\hline
\end{tabular}

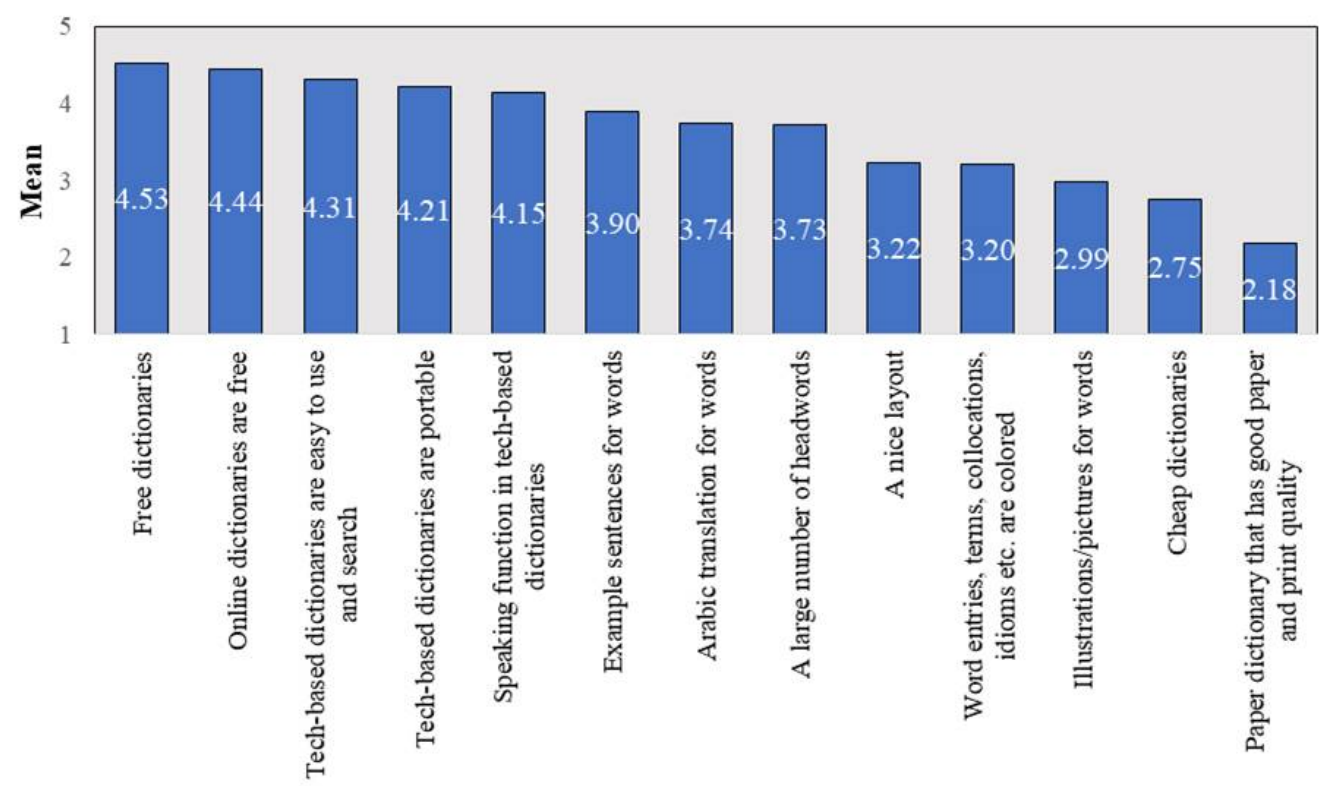

Figure 13. Likable features of dictionaries

\section{RQ7B. What features do English majors dislike most about dictionaries?}

The difficulty of search and use characteristic of paper dictionaries as well as their heavy weight and high thickness were the two features our subjects disliked most about dictionaries (means $=4.18$ and 4.17 respectively). In contrast, as we have seen in our answer to RQ7A relevant to the most likable dictionary features, this resentment for paper dictionaries was paralleled with a straightforward embracement of tech-based dictionaries. As such, our subjects reported that they never disliked tech-based dictionaries neither for lack of knowledge of how to use them (mean=1.31) nor out of dislike for technology in the first place (mean=1.19). Other features characteristic of some dictionaries that were repellent to our subjects included unclear explanations of some dictionary words (mean=4.11), small font size (mean=3.97) as well as outdated explanations of some words (mean $=3.94)$.

Again, as was the case with the likable features, our subjects' preferences on some aspects of the dictionary use strategy were clearly influenced by the dictionary features that they reported to dislike most. The following are 
the instances we spotted of the above-stated link between the dictionary features most dislikable to our subjects and their strategic preferences as to dictionary use:

\section{1). Difficulty of use and search in paper dictionaries}

- With relevance to means of dictionary ownership (RQ3), 'purchasing a paper dictionary myself', 'borrowing a paper dictionary from university library' as well as 'borrowing a paper dictionary from a friend' were among the very least common means of obtaining dictionary reported by our subjects. Unlike tech-based dictionaries which are easy to use and search, these three means of dictionary ownership involve the use of paper dictionaries which are far more difficult to use and search as we noted earlier.

- With relevance to frequency of dictionary consultation and dictionary type (RQ5B), our subjects reported looking up fewer words in paper dictionaries as opposed to the easier to use and search tech-based dictionaries.

- With relevance to dictionary format (RQ6A), paper dictionaries were rarely used and were very unpopular among our subjects. Compared to tech-based dictionaries, paper dictionaries are far more difficult to search and use.

\section{2). Heavyweight and high thickness of paper dictionaries}

- With relevance to means of dictionary ownership (RQ3), 'purchasing a paper dictionary myself', 'borrowing a paper dictionary from university library' as well as 'borrowing a paper dictionary from a friend' were among the very least common means of obtaining dictionary reported by our subjects. Unlike tech-based dictionaries which are clearly more portable and easier to carry, these three means of dictionary ownership involve the use of paper dictionaries which are less portable and more difficult to carry as we stressed earlier.

- With relevance to frequency of dictionary consultation and dictionary type (RQ5B), our subjects reported looking up fewer words in paper dictionaries as opposed to the more portable and easier to carry tech-based dictionaries.

- With relevance to dictionary format (RQ6A), paper dictionaries were rarely used and were very unpopular among our subjects. Compared to tech-based dictionaries, paper dictionaries are less portable and more difficult to carry due to their heavy weight and high thickness (see Table 17 and Figure 14).

Table 17. Dislikable features of dictionaries

\begin{tabular}{llll}
\hline Rank & Feature & Mean & SD \\
\hline $\mathbf{1}$ & Difficulty of use and search in paper dictionaries & 4.18 & 1.18 \\
$\mathbf{2}$ & Heavyweight and high thickness of paper dictionaries & 4.17 & 1.25 \\
$\mathbf{3}$ & Unclear explanations of some words & 4.11 & 1.15 \\
$\mathbf{4}$ & Small font size in some paper and tech-based dictionaries & 3.97 & 1.28 \\
$\mathbf{5}$ & Outdated explanations of some words & 3.94 & 1.28 \\
$\mathbf{6}$ & Expensive dictionaries & 3.93 & 1.31 \\
$\mathbf{7}$ & Poor paper and print quality of some paper dictionaries & 3.87 & 1.45 \\
$\mathbf{8}$ & Tech-based dictionaries because I don't know how to use them & 1.31 & .93 \\
$\mathbf{9}$ & Tech-based dictionaries because I don't like technology & 1.19 & .78 \\
\hline
\end{tabular}




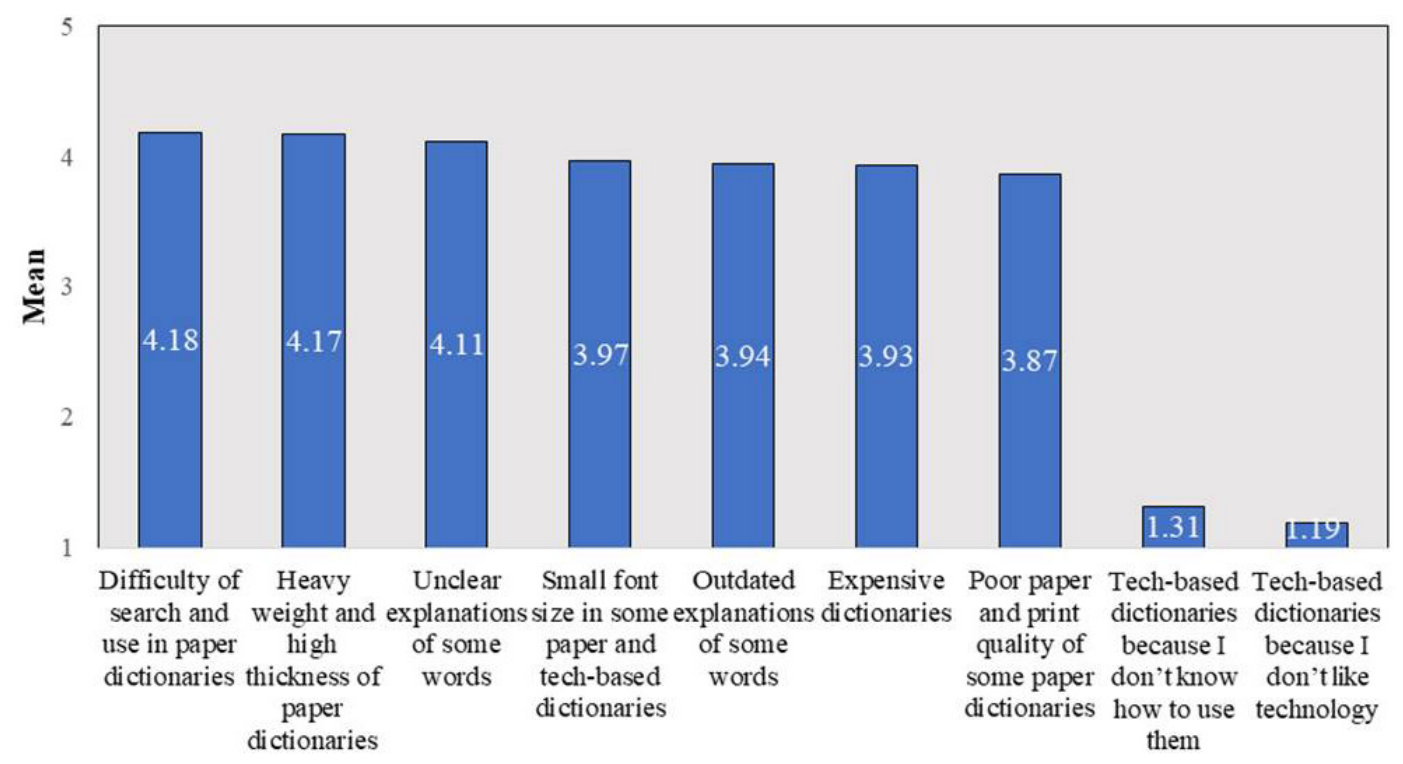

Figure 14. Dislikable features of dictionaries

\section{Conclusion}

This study surveyed the dictionary use sub-strategies of English majors in Saudi Arabia using a retrospective questionnaire adapted from the literature. Unlike other studies carried out in Saudi Arabia which were concerned with non-English major EFL learners, this study was particularly concerned with the dictionary use strategic preferences of learners majoring in English. Based on the findings of the study, we may conclude that the typical Saudi English-major learner when using the dictionary as a vocabulary learning strategy:

a. Mostly likes free dictionaries, the ease of use and search as well as portability of tech-based digital dictionaries

b. Mostly dislikes the difficulty of search and use in paper dictionaries as well as their heavy weight and high thickness

c. Uses the dictionary mainly to understand words he does not know when reading

d. Downloads dictionary apps to his phone from an application store or else goes online whenever he needs to consult a dictionary for a word

e. Uses the dictionary primarily at home

f. Uses the dictionary mainly for his basic English courses at university (i.e. listening, speaking, reading, writing)

g. Consults the dictionary no more than five times a day and tends to look up more words when consulting a tech-based digital dictionary than when using a paper dictionary

h. Favors the bilingual English-Arabic dictionary over other types (language-wise)

i. Favors dictionary apps and online dictionaries over other types (medium-wise)

j. Favors the ordinary dictionary over dictionaries of synonyms and/or collocations and idioms (content-wise)

k. Favors the English-Arabic dictionary for translation purposes over the Arabic-English or bilingualized English-English-Arabic dictionaries.

Thus, a major finding of this study is that tech-based digital dictionaries have clearly attracted Saudi English majors and it is for good reason: the numerous advantages they have over traditional paper dictionaries. With this sway of technology in the twenty-first century all over the world over seemingly all walks of life, we assume that EFL learners in general and English majors in particular from other cultural backgrounds have similar dictionary use patterns to our Saudi subjects. As such, based on the principle of learners' needs analysis largely cherished by dictionary making companies, we suggest that dictionary makers should consider putting more effort and, perhaps, more money into perfecting their tech-based versions of paper dictionaries so that they become more 
and more user-friendly. By the same token, curriculum and textbook designers, EFL school teachers and university English major instructors ought to find ways for making good use of this passion learners have for tech-based dictionaries either to further facilitate L2 learning and teaching tasks or else reinforce them. The findings of this study, the latter point, in particular, should hopefully generate some new lines of inquiry for ELT and/or TESOL researchers with interest in L2 learners' dictionary use research.

\section{References}

Al-Fuhaid, M. (2004). Vocabulary learning strategies: An empirical study of their use and evaluation by Saudi EFL learners majoring in English (Unpublished doctoral dissertation). Durham University, Durham, UK.

Alhaisoni, E. (2016). EFL teachers' and students' perceptions of dictionary use and preferences. International Journal of Linguistics, 8(6), 31-52. https://doi.org/10.5296/ijl.v8i6.10267

Al-Homoud, F. (2017). Dictionary use by Saudi EFL university preparatory program students. International Journal of Linguistics, 9(4), 15-27. https://doi.org/10.5296/ijl.v9i4.11103

Al-Qahtani, M. (2001). Vocabulary learning strategies (Unpublished master's thesis). University of Essex, Colchester, UK.

Al-Qahtani, M. (2005). The use of vocabulary learning strategies by EFL learners at three different educational levels (Unpublished doctoral dissertation). University of Essex, Colchester, UK.

Alyami, S. (2011). Vocabulary learning strategies of Saudi EFL majors of different gender, year and proficiency: Use and reasons for use (Unpublished doctoral dissertation). University of Essex, Colchester, UK.

Asher, C. (1999). Using dictionaries in the GCSE examination of modern foreign languages: Teachers' views and learners' performance. Studies in Modern Languages Education, 7, 59-67.

Béjoint, H. (1989). The teaching of dictionary use: Present state and future tasks. In F. Hausmann, O. Reichmann, H. Wiegand, \& L. Zgusta (Eds.), Dictionaries: An International Encyclopaedia of Lexicography (Vol. 1, pp. 208-215). Berlin: De Gruyter.

Bensoussan, M., Sim, D., \& Weiss, R. (1984). The effect of dictionary usage on EFL test performance compared with student and teacher attitudes and expectations. Reading in a Foreign Language, 2(2), 262-276.

Carstens, A. (1995). Language teaching and dictionary use: An overview. Lexikos, 5, 105-116.

Elola, I., Rodríguez-García, V., \& Winfrey, K. (2008). Dictionary use and vocabulary choices in L2 writing. ELIA, 8, 63-89.

Ezza, A., \& Saadeh, Z. (2011). Dictionary as a major resource for EFL course in pronunciation. World Journal of English Language, 1(1), 63-67. https://doi.org/10.5430/wjel.v1n1p63

Folse, K. (2004). Vocabulary myths: Applying second language research to classroom teaching. Michigan: University of Michigan Press. https://doi.org/10.3998/mpub.23925

Hadavi, M., \& Hashemi, Z. (2014). Comparative analysis of vocabulary learning strategies in learning English as a foreign language among freshmen and senior medical sciences students across different fields of study. Malaysian Journal of ELT Research, 10(2), 19-33.

Hamouda, A. (2013). A study of dictionary use by Saudi EFL students at Qassim University. Studies in English Language Teaching, 1(1), 227-257. https://doi.org/10.22158/selt.v1n1p227

Hartmann, R. (1989). Sociology of the dictionary user: Hypotheses and empirical studies. In F. Hausmann, O. Reichmann, H. Wiegand, \& L. Zgusta (Eds.), Dictionaries: An International Encyclopaedia of Lexicography (Vol. 1, pp. 102-111). Berlin: De Gruyter.

Huang, D. (2003). Taiwanese university English majors' beliefs about English dictionaries and their dictionary strategy use (Unpublished doctoral dissertation). The University of Texas at Austin, Austin, USA.

Kent, D. (2001). Korean university freshmen's dictionary use and perceptions regarding dictionaries. Korea TESOL Journal, 4(1), 73-92.

Laufer, B. (1990). Ease and difficulty in vocabulary learning: Some teaching implications. Foreign Language Annals, 23(2), 147-155. https://doi.org/10.1111/j.1944-9720.1990.tb00355.x

Luppescu, S., \& Day, R. (1993). Reading, dictionaries, and vocabulary learning. Language Learning, 43(2), 263-287. https://doi.org/10.1111/j.1467-1770.1992.tb00717.x 
McAlpine, J., \& Myles, J. (2003). Capturing phraseology in an online dictionary for advanced users of English as a second language: A response to user needs. System, 31(1), $71-84$. https://doi.org/10.1016/S0346-251X(02)00074-X

McCarthy, M. (1988). Some vocabulary patterns in conversation. In R. Carter, \& M. McCarthy (Eds.), Vocabulary and language teaching (pp. 181-200). London: Longman.

Mokhtar, A., Rawian, R., Yahaya, M., Abdullah, A., \& Mohamed, A. (2009). Vocabulary learning strategies of adult ESL learners. The English Teacher, 38, 133-144.

Nation, P. (1990). Teaching and learning vocabulary. New York: Newbury House.

Nesi, H., \& Haill, R. (2002). A study of dictionary use by international students at a British university. International Journal of Lexicography, 15(4), 277-305. https://doi.org/10.1093/ij1/15.4.277

Schmitt, N. (1997). Vocabulary learning strategies. In N. Schmitt, \& M. McCarthy (Eds.), Vocabulary: Description, acquisition, and pedagogy (pp. 199-227). Cambridge: Cambridge University Press.

Scholfield, P. (1982a). The role of bilingual dictionaries in ESL/EFL: A positive view. Guidelines, 4(1), 84-98.

Scholfield, P. (1982b). Using the English dictionary for comprehension. Tesol Quarterly, 16(2), $185-194$. https://doi.org/10.2307/3586791

Scholfield, P. (1997). Vocabulary reference works in foreign language learning. In N. Schmitt \& M. McCarthy (Eds.), Vocabulary: Description, acquisition, and pedagogy (pp. 279-302). Cambridge: Cambridge University Press.

Scholfield, P. (1999). Dictionary use in reception. International Journal of Lexicography, 12(1), 13-34. https://doi.org/10.1093/ij1/12.1.13

Şevik, M. (2014). University prep-school EFL learners' dictionary ownership and preferences. Procedia - Social and Behavioral Sciences, 158, 226-232. https://doi.org/10.1016/j.sbspro.2014.12.080

Summers, D. (1988). The role of dictionaries in language learning. In R. Carter, \& M. McCarthy (Eds.), Vocabulary and language teaching (pp. 111-125). London: Longman.

Tang, G. (1997). Pocket electronic dictionaries for second language learning: Help or hindrance? TESL Canada Journal, 15(1), 39-57. https://doi.org/10.18806/tesl.v15i1.691

Tseng, F. (2009). EFL students' Yahoo! online bilingual dictionary use behavior. English Language Teaching, 2(3), 98-108. https://doi.org/10.5539/elt.v2n3p98

Weschler, R., \& Pitts, C. (2000). An experiment using electronic dictionaries with EFL students. The Internet TESL Journal, 6(8), 56-67.

Wilkins, D. (1972). Linguistics in language teaching. London: Arnold.

\section{Notes}

Note 1. See section 3.1 Dictionary Use Strategy: Aspects and Sub-strategies for a detailed explanation as to why the researcher adopts using the term 'sub-strategies' instead of the literature-popular 'strategies'.

Note 2. Due to the considerable length of our survey questionnaire, as it included 115 items that covered all six aspects along with some others, we administered it on two phases (the first phase covered the dictionary related aspects whereas the second phase covered the word related aspects). In this study, we only report the findings of the first phase covering the dictionary related aspects. The findings related to the second phase will be reported in a separate study.

\section{Copyrights}

Copyright for this article is retained by the author(s), with first publication rights granted to the journal.

This is an open-access article distributed under the terms and conditions of the Creative Commons Attribution license (http://creativecommons.org/licenses/by/4.0/). 